\title{
Comparability of Pharmacodynamics Profiles with an Application to a Biosimilar Study
}

\author{
Jason JZ Liao ${ }^{1 *}$, Yifang $\mathrm{Li}^{2}$ and Xinhua Jiang ${ }^{3}$ \\ ${ }^{1}$ Merck \& Co., Inc, North Wales, PA 19454, USA \\ ${ }^{2}$ Novartis Pharmaceuticals Corporation, Cambridge, MA 02139, USA \\ ${ }^{3}$ Beijing University of Chemical Technology, Beijing, P.R. China
}

\begin{abstract}
It is often interest of comparing two pharmacodynamics (PD) profiles in drug development. Currently the common practice is borrowing the bioequivalence $(\mathrm{BE})$ rule in pharmacokinetics analysis for pharmacodynamics comparison in terms of the area under the effect curve (AUEC) of the pharmacodynamics profile. However, this may not be a feasible and sensitive enough approach since the bioequivalence approach is based on the summarized parameter of the pharmacodynamics profile rather than on directly comparison of the whole pharmacodynamics profile. In this paper, a simple but efficient and pragmatic pharmacodynamics comparability index is proposed to evaluate the comparability of pharmacodynamics profiles by comparing the whole pharmacodynamics profiles directly. Different biological products have different variability and the $\mathrm{CV} \%$ can be in a very large range. The PD comparability index can take account of the reference knowledge into consideration in assessment but the AUEC BE type approach ignores the reference variability. The good properties of the proposed approach are illustrated through simulated data and a real dataset.
\end{abstract}

Keywords: Comparability; Pharmacodynamics (PD) profile; PD comparability index; Power; Type I error

\section{Introduction}

In pharmaceutical development, pharmacodynamics (PD) markers are commonly used to understand the compound and speed up the drug development. For example, in developing biosimilars, the PD evaluations can provide critical and needed evidence in accumulating the totality of the evidence for the bio similarity between the proposed bio similar product and the reference product and providing convincing evidence for the extrapolation from one indication to other indications [1-5]. It is quite a challenge for demonstrating the PD comparability. Unlike pharmacokinetics $(\mathrm{PK})$, there is not much literature on $\mathrm{PD}$ comparison even for the small molecule. Most biologics usually have multiple indications. A couple of papers used the same bioequivalence (BE) rule in PK analysis for PD comparison in terms of the area under the effect curve (AUEC) of the PD profile [6,7]. Recently, FDA [8] issued guidance for conducting PD comparability study using the same BE rule. However, this may not be a feasible approach since the interpretation for AUEC used in PD studies is not the same as for PK studies since $\mathrm{PK}$ describes what the body does to the drug while PD describes what the drug does to the body. Since AUEC is summarized before comparing the PD profile directly, having the same AUEC for a PD profile does not necessarily imply the same PD profile. Note that the purpose of the comparability of pharmacodynamics is to compare the entire PD profile. Only one AUEC assessment for the PD profile may not be sensitive enough. To see this, Figure 1 shows two different PD profiles but having very similar AUEC values.

Here is the outline for the rest of the paper. In Section 2, a PD comparability index is proposed to compare the PD profile directly. In Section 3, simulation studies are used to compare the proposed PD comparability index with the AUEC BE approach to demonstrate the advantages of the proposed PD comparability index over the AUEC BE approach. A dataset is used to illustrate the method in Section 4. The summary is followed in Section 5.

\section{A PD Comparability Index}

There are many advanced statistical approaches in pharmacokinetics analysis besides the classical BE approach. For example, Dragalin, et al. [9] used Kullback-Leibler divergence for evaluating bioequivalence; Liao $[10,11]$ compared the pharmacokinetics profiles directly using a functional linear model. However, to conduct the PD comparison, the simple but efficient and pragmatic ideas for assessing the similarity of two dissolution profiles are borrowed here. Dissolution test in vitro is to ensure the drug product quality. The similarity of dissolution profiles is to ensure the product performance in the presence of a change such as scale-up, manufacturing site, component and composition, equipment and process, both immediaterelease (IR) and modified-release (MR) dosage formulations. To demonstrate the similarity of dissolution profiles, there are many proposed methods such as the simple graphics and summaries and many other advanced statistical methods. However, instead of using a more complicated statistical approach, FDA recommends and the industry commonly uses a relative non-sophisticated single similarity factor number $f$ to describe and quantify the difference between two dissolution profiles (Reference and Test) as:

$$
f_{2}=50 \times \log _{10}\left\{\left[1+\frac{1}{n} \sum_{t=1}^{n} w_{t}\left(R_{t}-T_{t}\right)^{2}\right]^{-0.5} \times 100\right\}
$$

Where $n$ is the total number of dissolution time points and $w_{t}$ is the optional weight factor. The transformation is to scale the factor into $(0$, $100)$. A value $f_{2}=100$ means the two dissolution profiles are exactly the same. A higher $f_{2}$ number means a better similarity for the dissolution profiles. The similarity of dissolution profiles is claimed if the lower $95 \%$ confidence limit of $f_{2}>50$, which is about $10 \%$ dissolution profile difference/changes [12-15].

*Corresponding author: Liao JJZ, Merck \& Co., Inc, North Wales, PA 19454, P.R. China, Tel: 7175317178; E-mail: a4statistics@gmail.com

Received April 20, 2017; Accepted April 25, 2017; Published April 28, 2017

Citation: Liao JJZ, Li Y, Jiang X (2017) Comparability of Pharmacodynamics Profiles with an Application to a Biosimilar Study. J Biom Biostat 8: 345. doi: 10.4172/2155-6180.1000345

Copyright: @ 2017 Liao JJZ, et al. This is an open-access article distributed under the terms of the Creative Commons Attribution License, which permits unrestricted use, distribution, and reproduction in any medium, provided the original author and source are credited. 


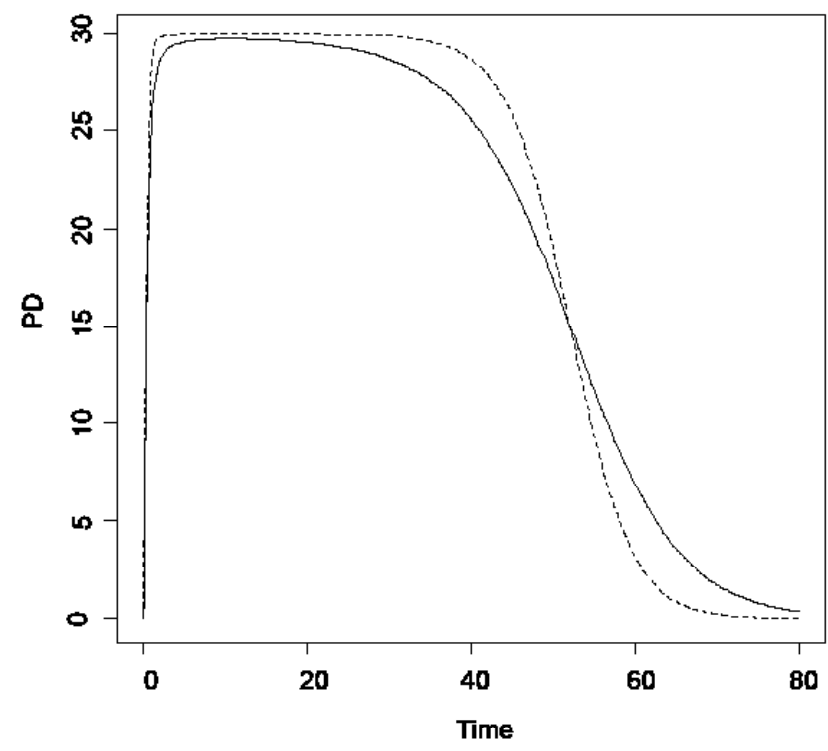

Figure 1: Illustration of two different PD profiles but having the similar AUEC values.

To mimic the evaluation for the similarity of dissolution profiles with a relatively non-sophisticated and pragmatic approach, a PD comparability index to assess the comparability of PD profiles is proposed as follows.

$$
f_{P D}=\frac{\min \left(R_{\max }^{R}-R_{\min }^{R}, R_{\max }^{T}-R_{\min }^{T}\right)}{\max \left(R_{\max }^{R}-R_{\min }^{R}, R_{\max }^{T}-R_{\min }^{T}\right)+\sqrt{\frac{1}{n} \sum_{j=1}^{n} w_{j}\left(\mu_{j}^{R}-\mu_{j}^{T}\right)^{2}}}
$$

where $n$ is the total number of time points in the PD profile; $\mu_{j}^{R}$ and $\mu_{j}^{T}$ are the PD responses at time $t_{j}$ for the reference and the test product, respectively; $w_{j}$ is the optional weight. $R_{\max }^{R}\left(R_{\min }^{R}\right)$ and $R_{\max }^{T}\left(R_{\min }^{T}\right)$ are the maximum (minimum) profile response for the reference and the test product, respectively. This PD comparability index is within $(0$, 1 ), where 1 indicates the $100 \%$ comparability, i.e., the identical PD profile. A higher $f_{P D}$ number means a better comparability for the two PD profiles. The PD comparability is claimed if 1) The approximate lower $95 \%$ confidence limit of the PD comparability index $f_{P D}$ for the test against the reference is greater than a value $\delta_{0}$; and 2) The point estimate of the index $f_{P D}$ for the test against the reference is greater than a value $\delta_{1}$.

The two boundaries $\delta_{0}$ and $\delta_{1}$ used in the PD comparability index approach can be determined beforehand with the consensus from the regulatory authorities. The first condition $\delta_{0}$ in the acceptance criteria takes account of the reference variability and is the acceptable limit. One way to decide $\delta_{0}$ is to use the reference against the reference as the base to make a comparison between the test and the reference product. Following Chow, et al. [16] in defining the biosimilarity limit, a discounting (say, 0.9) of the approximate lower 95\% confidence limit of the PD comparability index using only the reference against the reference information can be provided as a way of selecting the $\delta_{0}$, the threshold for accepting the test against the reference. The second condition in the acceptance criteria is to ensure no major PD profile difference for the test product passed the comparability due to a large variability for the reference product $[17,18]$ which is usually the case for a biological product. Toward this, a value of $\delta_{1}=0.9$, which is about $10 \%$ of the mean difference could be a reasonable value for use.
Note that in assessing the similarity of the dissolution profiles, a fixed boundary 50 in the acceptance criterion was used as the lower boundary of $f_{2}$ and the amount 50 is the $f_{2}$ value when there is a $10 \%$ dissolution profile difference. To mimic this and simplify the acceptance criteria, then $\delta_{0}$ can also be set as a fixed value regardless of the reference variability, say, 0.77 , which accounts for about $30 \%$ $\mathrm{PD}$ profile difference/changes relative to the response range when the maximum (minimum) response is the same for both the test product and the reference product. The third choice of the $\delta_{0}$ can also be set as the maximum of a fixed value and the lower confidence limit of the PD comparability index from the reference against the reference itself. In summary, to select an appropriate $\delta_{0}$ in the first condition, three methods can be used.

1) For simplicity, $\delta_{0}$ can be set at a fixed value regardless of the reference variability. For example, $\delta_{0}$ could be set at 0.77 to account for $30 \%$ PD profile difference/changes when the maximum (minimum) response is the same for both the test biologics and the reference product.

2) To consider the reference variability, $\delta_{0}$ can be set at the lower boundary of the $95 \%$ one-sided confidence interval of the reference against reference multiplied by a discount constant factor $c(0<c<1)$ say, $c=0.9$.

3) $\delta_{0}$ can be set as the maximum of the values obtained from the previous two methods.

To estimate the value of $f_{P D}$ in eqn. (2), the corresponding parameters can be obtained by fitting a model such as the Emax model or the extended Emax model [19], or from a smooth curve of the PD profile using a non-parametric method such as the smooth spline and the confidence bands of the smooth spline can be constructed using the method [20] in which can be used to derive the lower $95 \%$ confidence limit of $f_{P D}$ as follows

$$
\frac{\min \left(y_{\max }^{R}-y_{\min }^{R}, y_{\text {max }}^{T}-y_{\text {min }}^{T}\right)}{\max \left(y_{\max }^{R}-y_{\min }^{R}, y_{\text {max }}^{T}-y_{\min }^{T}\right)+\sqrt{\max \left\{\frac{1}{n} \sum_{j=1}^{n} w_{j}\left(y_{j}^{R}-y_{j}^{T} \pm z_{0.975} \sqrt{S_{R}^{2}+S_{T}^{2}}\right)^{2}\right\}}}
$$

Where $S_{k}^{2}, k=R, T$ is the residual variance for the reference and the test product, respectively, $y_{j}^{R}$ and $y_{j}^{T}$ are the predicted PD responses at time $t_{j}$ for the reference and the test product, respectively; $y_{\max }^{R}\left(y_{\min }^{R}\right)$ and $y_{\max }^{T}\left(y_{\min }^{T}\right)$ are the predicted maximum (minimum) profile response for the reference and the test product, respectively. If possible, the pharmacokinetics and pharmacodynamics (PK/PD) is preferably evaluated in the same subjects to obtain information on the PK-PD relationship. To construct the confidence interval of $f_{P D}$ for reference against the reference, a bootstrap method is recommended to construct the approximate lower $95 \%$ confidence limit. The bootstrapping is to resample from the subjects with replacement twice to form two data sets with each having sample size $n$, then to extract the corresponding responses to refit the PD profile curves using smoothing splines and compute the confidence interval limit using eqn. (3) based on the estimated results of the two resampled data sets. The bootstrapping steps were repeated for $\mathrm{N}$ times and the lower $2.5 \%$ percentile of the PD comparability index is then used as the approximate $95 \%$ lower confidence limit.

\section{Simulation Studies}

In this section, the simulation study is used to compare the performance of the BE approach using the AUEC under the PD profile and the proposed PD comparability index in terms of the type I error 
and power. The data were generated from the PD profile. The following two PD profiles are considered in this simulation study:

$$
P D_{1}=30-\frac{30}{1+[200 \times(\exp \{-0.09 t\}-\exp \{-0.1 t\})]^{2}}
$$

and

$$
P D_{2}=30-\frac{30}{1+[400 \times(\exp \{-0.09 t\}-\exp \{-0.1 t\})]^{2}},
$$

Where $P D_{1}$ is considered as the reference product. When the PD profiles from the reference and the test product are different as shown in Figure 2, the type I errors of the two approaches are compared. When the test product also takes the same PD profile of the reference, the powers of the two approaches are compared. It is a well-known fact that the variability varies from the time points and thus, a heterogeneity error term is used in generating the data. In detail, the PD is evaluated at the following time points: $0,0.25,0.50,1,2,3,4,5,6,8,12,18,24$, $30,36,42,48,60$, and 72 hours after dosing with a heterogeneity error $0.4 \exp (0.1 y) \times \varepsilon$, where $y$ is the true $\mathrm{PD}$ value and $\varepsilon$ is from a standard normal distribution. Three different sample sizes $n=30,40$, and 60 for each product were used for the simulation. At each setting, the same experiment was repeated 1000 times.

To determine the comparability between the reference and the test product, the AUEC BE approach and the proposed PD comparability

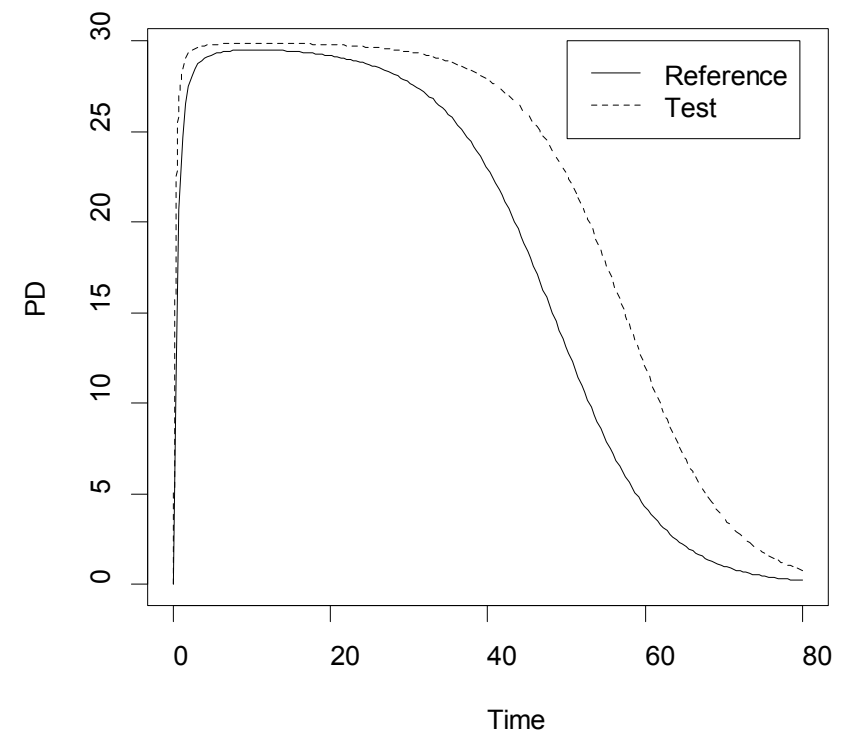

Figure 2: Simulation setting for evaluating the type I error when the two PD profiles are different. The solid line is for the reference and the dotted line is for the test product. index approach were used. For the AUEC BE approach, the BE 0.8-1.25 criterion was used. For the proposed PD comparability index approach, the cubic smoothing spline was used for estimating the PD curve. The comparability index and the $95 \%$ confidence interval limit of reference against test were estimated using eqns. (2) and (3), where the optional weight was not used. In the PD comparability index approach criteria, two values of $\delta_{0}$ were used: $\delta_{1}=0.9$ for $10 \%$ mean difference and $\delta_{1}=0.83$ for $20 \%$ mean difference. For $\delta_{0}$ in the first criterion, all three different methods were used in this simulation. For the reasonable fixed constant, two constant values were considered: 0.77 (accounting for 30\% PD difference), and 0.8 (accounting for 25\% PD difference). The reference against reference confidence interval boundary was computed using the bootstrap method. For each method, the bootstrapping was done 1000 times. The rate of passing PD comparability from the 1000 simulations is shown in Table 1.

When there is no PD profile difference, Table 1 indicates that both AUEC BE type approach and the proposed PD comparability index approach have good powers. However, when the reference curve and the test curve are different given as $P D_{1}$ and $P D_{2}$ shown in Figure 2, respectively, the AUEC BE criterion tends to claim them to be comparable and leads to a large type I error. However, the newly proposed PD comparability index approach can give satisfactory results when $\delta_{0}$ and $\delta_{1}$ are chosen properly, with a well-controlled type I error under or around 0.05. Larger $\delta$, has a better control on the type I error. Note that the choice of $\delta_{0}$ as the maximum of a fixed and a reference variability based has exactly the same results as the reference variability based because in the simulation settings, the "max" always picks up the "90ref" value as the "90ref" is always greater than the fixed boundary. This may not be the case when reference variability is large, which can be seen in the illustration example. To achieve a better control of the type I error, the maximum value of a fixed value and the reference variability dependent value is recommended. The simulation results also show that the type I error is also better controlled when the sample size is relatively large, as expected.

It is a well-known fact that the sampling time points may have a big effect in the assessment. Toward this, two more different sampling schemes were used in the simulation studies. One was with less sampling time points: $0,0.50,2,4,8,12,18,24,36,48,60$, and 72 . The other was with much less sampling time points: $0,0.50,4,8,12,18$, 48 , and 72. For these two extra sampling schemes, the AUEC BE type approach and the PD comparability index approach generated similar results (not shown here) and had similar conclusions in terms of the type I error and the power as shown in Table 1.

In summary, the PD comparability index compares the whole PD profile directly and provides more sensitive and the type I error controlled assessment comparing to the AUEC BE type approach. The

\begin{tabular}{|c|c|c|c|c|c|c|c|c|c|c|c|c|}
\hline & \multirow{3}{*}{$\begin{array}{c}\text { Sample Size } \\
\text { Per Arm }\end{array}$} & \multirow[t]{3}{*}{ AUEC BE } & \multirow[b]{3}{*}{$\delta_{0}$} & \multicolumn{9}{|c|}{ PD Comparability Index } \\
\hline & & & & \multicolumn{3}{|c|}{$\delta_{1}=0.9$} & \multicolumn{3}{|c|}{$\delta_{1}=0.83$} & \multicolumn{3}{|c|}{$\delta_{1}=0.83$} \\
\hline & & & & 0.77 & 90Ref & Max & 0.77 & 90Ref & Max & 0.8 & 90Ref & Max \\
\hline \multirow[t]{3}{*}{ Power } & $N=30$ & 1 & & 1 & 1 & 1 & 1 & 1 & 1 & 1 & 1 & 1 \\
\hline & $\mathrm{N}=40$ & 1 & & 1 & 1 & 1 & 1 & 1 & 1 & 1 & 1 & 1 \\
\hline & $\mathrm{N}=60$ & 1 & & 1 & 1 & 1 & 1 & 1 & 1 & 1 & 1 & 1 \\
\hline \multirow[t]{3}{*}{ Type I Error } & $N=30$ & 1 & & 0 & 0 & 0 & 1 & 0.044 & 0.044 & 0.295 & 0.044 & 0.044 \\
\hline & $\mathrm{N}=40$ & 1 & & 0 & 0 & 0 & 0.999 & 0.022 & 0.022 & 0.232 & 0.022 & 0.022 \\
\hline & $\mathrm{N}=60$ & 1 & & 0 & 0 & 0 & 1 & 0.004 & 0.004 & 0.178 & 0.004 & 0.004 \\
\hline
\end{tabular}
proposed PD comparability index can take account of the reference

Table 1: Rate of passing PD comparability from simulation studies. 
variability and reference knowledge into consideration while the AUEC BE type approach does not.

\section{An Illustration}

Consider a study for a proposed biosimilar cancer drug to a marketed innovator cancer drug. A parallel-design study was conducted to assess the PK/PD comparability of the biosimilar biologics to the marketed innovator at two different dose levels $0.1 \mathrm{mg} / \mathrm{kg}$ and $0.3 \mathrm{mg} /$ $\mathrm{kg}$ [21-23]. At the dose level $0.1 \mathrm{mg} / \mathrm{kg}$, twenty eight (28) subjects were allocated to both the marketed innovator and the proposed biosimilar biologics. The blood for pharmacokinetics was drawn at baseline and 4 , $10,24,34,48,72$, and 96 hours after drug administration; and the PD was evaluated at the baseline and 4,24, 48, 72, and 96 hours after drug administration. However, there was only one reading for one subject for both the biosimilar biologics and the reference product, and thus, this subject were not included in this evaluation. The raw data for the PD endpoint absolute neutrophil count (Neut) are plotted against the time to generate the PD profile in Figure 3, in which much higher variability is shown in the middle range of the PD profile. Before the formal analyses, the summary statistics in terms of the mean and the standard deviation at each time point are listed in Table 2. The comparison for the PD endpoint absolute neutrophil count (Neut) at each time point was conducted. The $95 \%$ confidence interval of the difference between the biosimilar and the reference and the p-value testing the mean difference between the test and reference are also listed in Table 2 , which indicates that there are no statistically significant differences at all the sampled time points and the confidence interval indicates the two products are comparable at all the sampled time points.

As the first formal analysis, the AUEC of the PD profile for each subject was calculated and the BE approach was used to assess the PD comparability of the proposed biosimilar and the reference. The estimated GMR of the biosimilar against the reference is 0.897 with the $90 \%$ confidence interval $(0.875,1.026)$. Thus, the proposed biosimilar and the reference are bioequivalence in terms of area under the PD profile using the $\mathrm{BE}$ criteria. Since the goal of the PD comparability is to compare the whole PD profile, it may not be good enough to just compare the PD responses at each observed time points as shown in Table 2. For this purpose, the proposed PD comparability index defined in eqn. (2) was also used. The data are plotted together with the fitted PD interpolation profiles for both the reference and the test in Figure 4.

To quantify the similarity of PD profiles between the test and the innovator product, the proposed PD comparability index was calculated. The estimated PD comparability index is 0.975 , and the lower $95 \%$ confidence interval limit is 0.767 . To compute the reference against the reference confidence interval, 1000 bootstrap replications were used, and in each bootstrap replication, the reference data set was resampled twice to form two data sets. The average of the 1000 lower confidence interval limits is 0.723 , which indicates that there is a considerable variability for the reference product itself and this value 0.723 can account for $38 \%$ PD profile difference/changes. The results
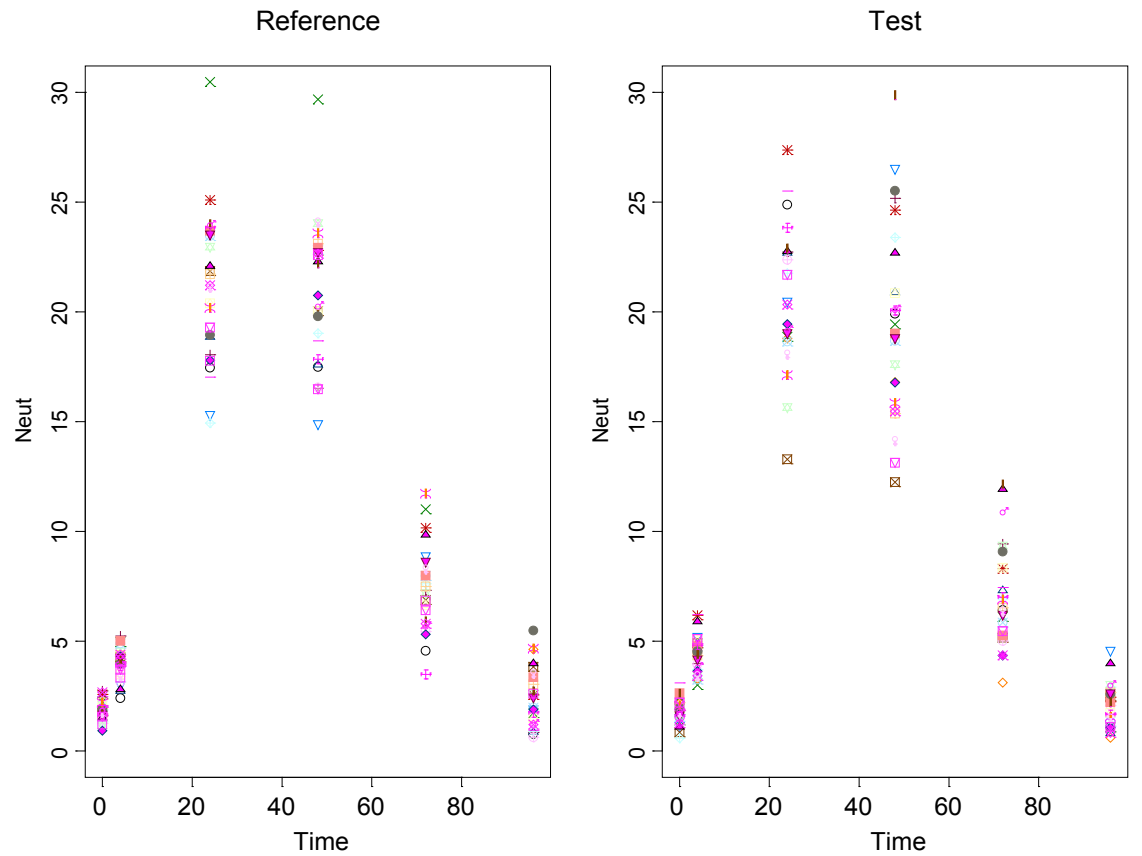

Figure 3: Raw data plot for the PD profile.

\begin{tabular}{|c|c|c|c|c|c|c|c|}
\hline \multicolumn{2}{|c|}{ Time (hr) } & \multicolumn{6}{|c|}{ Mean (Standard Deviation) } \\
\hline & & 0 & 4 & 24 & 48 & 72 & 96 \\
\hline \multirow[t]{4}{*}{ PD } & Reference (R) & $1.837(0.463)$ & $3.919(0.750)$ & $20.960(3.455)$ & $20.793(3.223)$ & 7.375 (1.995) & $2.353(1.344)$ \\
\hline & Test $(T)$ & $1.763(0.572)$ & $4.368(0.894)$ & $20.753(3.272)$ & $19.728(4.329)$ & $7.124(2.288)$ & $2.030(0.983)$ \\
\hline & $95 \% \mathrm{Cl}(\mathrm{T}-\mathrm{R})$ & $(-0.207,0.355)$ & $(-0.915,0.016)$ & $(-1.734,2.147)$ & $(-1.035,3.164)$ & $(-0.975,1.475)$ & $(-0.329,0.973)$ \\
\hline & $p$-value ${ }^{*}$ & 0.599 & 0.058 & 0.831 & 0.313 & 0.683 & 0.325 \\
\hline
\end{tabular}

Table 2: Summary statistics at each time point. 


\begin{tabular}{|c|c|c|c|c|c|c|c|c|c|c|}
\hline \multirow[t]{3}{*}{ AUEC BE } & & \multicolumn{9}{|c|}{ PD Comparability Index } \\
\hline & & \multicolumn{3}{|c|}{$\delta_{1}=0.9$} & \multicolumn{3}{|c|}{$\delta_{1}=0.9$} & \multicolumn{3}{|c|}{$\delta_{1}=0.83$} \\
\hline & $\delta_{0}$ & 0.74 & 90Ref & Max & 0.77 & 90Ref & Max & 0.77 & 90Ref & Max \\
\hline Pass & & Pass & Pass & Pass & Fail & Pass & Fail & Fail & Pass & Fail \\
\hline
\end{tabular}

Table 3: Data analysis results for the example dataset.

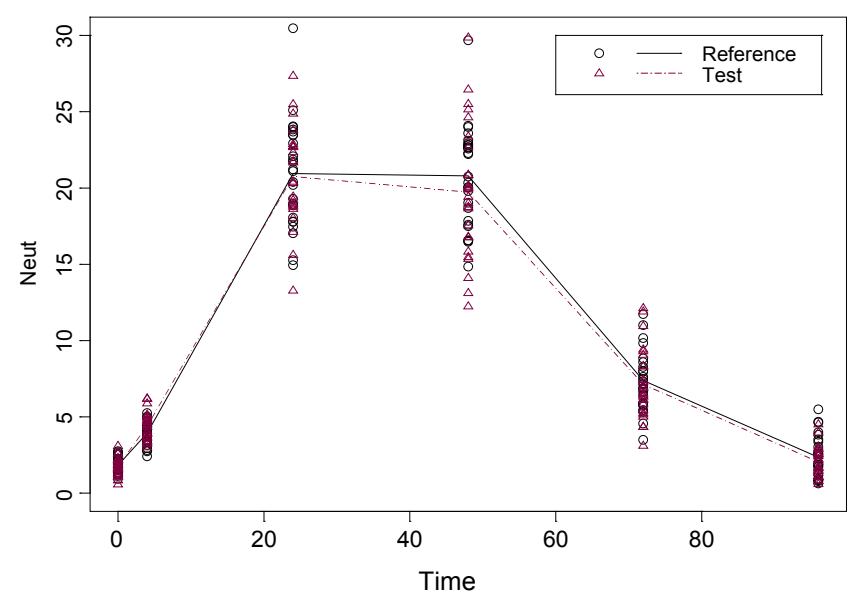

Figure 4: Overlaid raw data with estimated PD interpolation profiles.

of the PD comparability index approach using different $\delta_{0}$ and $\delta_{1}$ are summarized in Table 3 .

Table 3 indicates that the conclusion depends on the choice of $\delta_{0}$ and $\delta_{1}$. The estimated PD comparability index of the test against the reference is large, and greater than both the selected $\delta_{1}$ values, thus the $\delta_{1}$ criteria passed. Since the estimated lower confidence interval limit for the PD comparability index of the test against the reference is 0.767 but the estimated confidence interval lower limit for the PD comparability index of the reference against the reference is 0.723 , thus, the maximum value always picks up the constant $\delta_{0}$ as 0.74 , or 0.77. If only the reference against reference PD comparability index information is used, then PD comparability conclusion can be claimed on all parameter choices. The choice of a fixed boundary or the maximum of the fixed and the reference variability based (this leads to the same as the fixed boundary in this example) will impact the final conclusion. The PD comparability only passes for the choice of 0.74 but fails the choice of 0.77 .

\section{Summary}

Although more advanced statistical techniques can be developed, a simple but efficient and pragmatic PD comparability index $f_{P D}$ was proposed to demonstrate the comparability of PD profiles between the test biologics and the reference product directly in this paper. This PD comparability index compares the two PD profiles directly while the AUEC is only a summary of the PD profile which may be not sensitive enough. Much more confident conclusion can be drawn using this PD comparability index than using the only summarized parameter AUEC of the PD profile. Different biological products have different variability and the $\mathrm{CV} \%$ can be in a very large range. The $\mathrm{PD}$ comparability index can take account of the reference knowledge into consideration in assessment but the AUEC BE type approach ignores the reference variability.

The two boundaries $\delta_{0}$ and $\delta_{1}$ used in the PD comparability index approach can be determined beforehand with the consensus from the regulatory authorities. Different choices of the acceptance criterion for the PD comparability were discussed in this paper. One of the choices for $\delta_{0}$ was based on the comparison of the reference against the reference itself. The PD comparability index of the test against the reference is compared to the PD comparability index of the reference against itself. In this case, the variability of the reference product plays an important role and the acceptance boundary is a reference-scaled based. With that said the acceptance criteria may vary from product to product. A simpler acceptance criterion was also proposed. For example, the approximate lower 95\% confidence limit of the PD comparability index for the test against the reference can be compared to a fixed value, say, 0.77 , which allows for $30 \% \mathrm{PD}$ profile changes when the maximum (minimum) response is the same for both the test and the reference product. This acceptance boundary is for all reference products regardless the reference variability, thus, it is not a referencescaled based but is used as the presumed most tolerable difference. Other fixed value after the discussion with regulatory authorities can also be used. However, from the simulation and the example, in order to achieve a better control of type I error, the maximum of the reference scaled boundary and a fixed boundary is recommended.

As evidenced in Figure 1, the sampling time points and the number of time points can be very critical toward the final conclusion of the PD comparability assessment. The influence factors can from the PK mechanism, the PD mechanism, and the shape of the PD time profile. It is the goal to design a small but yet very efficient PD comparability study and this can be very challenging.

\section{References}

1. Food and Drug Administration (FDA) of USA (2012) Guidance for industry: Scientific considerations in demonstrating bio similarity to a reference product. Washington DC

2. Liao JJZ, Heyse JF (2011) Biosimilarity for Follow-on Biologics. Statistics in Biopharmaceuticals Research 3: 445-455

3. Saurwein-Teissl M, Lang-Salchner M and Kirchlechner T (2011) Global Biosimilar Development. Global Forum 3: 39-43.

4. World Health Organization (WHO) (2009) Guidelines on evaluation of similar bio therapeutic products (SBPs). Geneva.

5. EMA Committee for Medicinal Products for Human Use (CHMP) (2005) Guideline on similar biological medicinal products. London.

6. Varki R (2009) A glycosylated recombinant human granulocyte colony stimulating factor produced in a novel protein production system (AVI-014) in healthy subjects: a first-in human, single dose, controlled study. BMC Clinical Pharmacology 9: 1-8

7. Waller CF, Bronchud M, Mair S, Challand R (2010) Comparison of the pharmacodynamics profiles of a biosimilar filgrastim and Amgen filgrastim: results from a randomized, phase I trial. Ann Hematol 89: 971-978.

8. Food and Drug Administration (FDA) of USA (2014) Guidance for industry Clinical Pharmacology Data to Support a Demonstration of Biosimilarity to a Reference Product. Washington.

9. Dragalin V, Fedorov V, Patterson S, Jones B (2003) Kullback-Leibler divergence for evaluating bioequivalence. Statistics in Medicine 22: 913-930.

10. Liao JJZ (2003) A Functional Linear Model for Comparing Two Pharmacokinetics Profiles. J of Statistical Planning and Inference 116: 521-535.

11. Liao JJZ (2005) Comparing the Concentration Curves Directly in a Pharmacokinetics, Bioavailability/Bioequivalence Study. Statistics in Medicine 24: 883-891. 
Citation: Liao JJZ, Li Y, Jiang X (2017) Comparability of Pharmacodynamics Profiles with an Application to a Biosimilar Study. J Biom Biostat 8: 345. doi: $10.4172 / 2155-6180.1000345$

Page 6 of 6

12. Kumar P, Mishra B (2008) Comparison of in-vitro dissolution profiles-an overview. The Pharma Review: 94-100.

13. Tsong Y, Sathe PM, Shah VP (2003) In Vitro dissolution profile comparison. Encyclopedia of Biopharmaceutical Statistics, Marcel Dekker.

14. O'Hara T, Dunne A, Butler J, Devane J (1998) A review of methods used to compare dissolution profile data. Pharmaceutical Science \& Technology Today 1: $214-223$.

15. Shah VP, Tsong Y, Sathe P, Liu JP (1998) In Vitro dissolution profile comparison-statistics and analysis of the similarity factor, $f_{2}$. Pharmaceutical Research 15: 889-896.

16. Chow SC, Yang LY, Starr A, Chiu ST (2013) Statistical methods for assessing interchangeability of biosimilars. Statistics in Medicine 32: 442-448.

17. Haidar SH (2007) Bioequivalence approaches for highly viable drugs and drug products. Pharmaceutical Research 25: 237-241.
18. Haidar SH (2008) Evaluation of scaling approach for the bioequivalence of highly variable drugs. The AAPS Journal 10: 450-454.

19. Sarkar CA, Lauffenburger DA (2003) Cell-level PK model of granulocyte colony-stimulating factor: implications for ligand lifetime and potency in vivo. Molecular Pharmacology 63: 147-158.

20. Hastie TJ, Tibshirani RJ (1990) Generalized Additive Models. Chapman and Hall, London.

21. Food and Drug Administration (FDA) of USA (2001) Guidance for industry: Statistical approaches to establishing bioequivalence. Washington.

22. Food and Drug Administration (FDA) of USA (2012) Guidance for industry: Quality considerations in demonstrating bio similarity to a reference product. Washington.

23. Food and Drug Administration (FDA) of USA (2012) Guidance for industry: Biosimilars: Questions and answers regarding implementation of the biologics price competition and innovation act of 2009. Washington. 